Magnetohydrdynamic Flow Past a Porous

Rotating Disk in a Circular Magnetic Field

S. Kishore Kumar

William I. Thacker

Layne T. Watson

TR 87-2 


\title{
MAGNETOHYDRODYNAMIC FLOW PAST A POROUS ROTATING DISK IN A CIRCULAR MAGNETIC FIELD
}

\author{
S. Kishore Kumart \\ Director's Unit \\ National Aeronautical Laboratory \\ Bangalore, India 560017 \\ William I. Thacker \\ Computer Science \\ Winthrop College \\ Rock Hill, South Carolina 29733 \\ Layne T. Watson $\ddagger$ \\ Department of Computer Science \\ Virginia Polytechnic Institute and State University \\ Blacksburg, Virginia 24061
}

\begin{abstract}
This paper studies the effects of a circular magnetic field on the flow of a conducting fuid about a porous rotating disk. Using modern quasi-Newton and globally convergent homotopy methods, numerical solutions are obtained for a wide range of magnetic field strengths, suction and injection velocities, and Alfven and disk speeds. Results are presented graphically in terms of three nondimensional parameters. There is excellent agreement with previous work and asymptotic formulas.
\end{abstract}

\section{INTRODUCTION.}

The flow past a rotating disk in a viscous fluid has received considerable attention in the past few decades. Von Karmen [1] first obtained a solution for this problem. Later Cochran [2] obtained a more accurate solution by numerical integration of the governing equations. The effects of suction and injection at the disk surface were studied later by Stuart [3], Sparrow and Gregg [4], Kuiken [5], and Ackroyd [6].

The rotating disk problem in the presence of an axial magnetic field was studied by Sparrow and Cess [7], Rizvi [8] (whose mathematical formulation and numerical solutions are incorrect), and Pande [9]. Pao [10] studied the flow of a viscous electrically conducting fiuid past a rotating disk with a circular magnetic field at the disk surface.

In the present investigation we study the effects of surface suction and injection on the flow past a rotating disk in the presence of a circular magnetic field. The governing equations are similar to those derived by $\mathrm{Pao}$ [10]. Here we assume that there is no magnetic field in the fluid far from the disk, and also, that there is a field in the tangential direction in the boundary layer generated by external means within the disk itself. Such a field may have application in the shielding of a rotating body from excessive heating [10].

$\$$ The work of this author was supported by a fellowship from CSIR, Delhi.

$\$$ The work of this author was supported in part by Air Force Office of Scientific Research Grant 85-0250. 
The governing partial differential equations of the problem are reduced to nonlinear ordinary differential equations using similarity transformations. The system is then solved numerically using modern quasi-Newton and homotopy methods. The flow depends on the nondimensional parameters $\alpha$ (magnetic Prandtl number), $\beta$ (ratio of Alfven speed to the disk speed), and $A$ (suction or injection parameter).

\section{FORMULATION OF THE PROBLEM.}

The governing equations in cylindrical polar coordinates $(r, \theta, z)$ for an incompressible, viscous, electrically conducting fluid in axisymmetric steady motion [8] are

$$
\begin{aligned}
u \frac{\partial u}{\partial r}+w \frac{\partial u}{\partial z}-\frac{v^{2}}{r}+\frac{\partial P}{\partial r}-f \frac{\partial f}{\partial r}-h \frac{\partial f}{\partial z}+\frac{g^{2}}{r}-\nu\left(\nabla^{2} u-\frac{u}{r^{2}}\right) & =0 \\
u\left(\frac{\partial v}{\partial r}+\frac{v}{r}\right)+w \frac{\partial v}{\partial z}-f\left(\frac{\partial g}{\partial r}+\frac{g}{r}\right)-h \frac{\partial g}{\partial z}-\nu\left(\nabla^{2} v-\frac{v}{r^{2}}\right) & =0 \\
u \frac{\partial w}{\partial r}+w \frac{\partial w}{\partial z}+\frac{\partial P}{\partial z}-f \frac{\partial h}{\partial r}-h \frac{\partial h}{\partial z}-\nu \nabla^{2} w & =0 \\
\frac{\partial}{\partial r}(r u)+\frac{\partial}{\partial z}(r w) & =0 \\
\frac{\partial}{\partial r}(r f)+\frac{\partial}{\partial z}(r h) & =0 \\
\frac{\partial}{\partial r}(v f-u g)+\frac{\partial}{\partial z}(v h-w g)+\eta\left(\nabla^{2} g-\frac{g}{r^{2}}\right) & =0 \\
(u h-w f)-\eta\left(\frac{\partial h}{\partial r}-\frac{\partial f}{\partial z}\right) & =0
\end{aligned}
$$

where

$$
\nabla^{2}=\frac{\partial^{2}}{\partial r^{2}}+\frac{1}{r} \frac{\partial}{\partial r}+\frac{\partial^{2}}{\partial z^{2}}
$$

and $u, v, w$ and $f, g, h$ are the components of velocity and normalized magnetic field strength respectively in the $r, \theta, z$ directions respectively. $\nu$ is the kinematic viscosity, $\eta=1 / \mu \sigma$ is the magnetic diffusivity, $\mu$ is the magnetic permeability and $\sigma$ is the electrical conductivity. Here

$$
(f, g, h)=\left(f_{1}, g_{1}, h_{1}\right)(\mu / \rho)^{1 / 2} \text { and } P=\left(f^{2}+g^{2}+h^{2}\right) / 2+p / \rho+\chi,
$$

where $f_{1}, g_{1}, h_{1}$ are the components of the magnetic field, $p$ is the fluid pressure, $\rho$ is the uniform density and $\chi$ is the potential for a unit mass of the conservative body forces. It is assumed that $\nu$, $\sigma$ and $\mu$ are constants and the net charge density is zero.

\section{SIMILARITY SOLUTION AND BOUNDARY CONDITIONS.}

The disk suriace is in the plane $z=0$ and rotates about the $z$-axis with constant angular velocity $\omega$. An axial electric current of uniform current density $J_{0}$ is imposed at the disk surface. Equiralently, a tangential magnetic field component $g=\Omega r$ is imposed at the disk surface with $\Omega=(\mu / \rho)^{1 / 2}\left(J_{0} / 2\right)$ a constant, and $f$ and $h$ are zero. 
Using the following similarity transformations

$$
\begin{array}{lll}
u=\omega r m^{\prime}(\zeta), & w=-2(\nu \omega)^{1 / 2} m(\varsigma), & P=\nu \omega S(\varsigma), \\
v=\omega r G(\varsigma), & g=\Omega r M(\zeta), & \zeta=z(\omega / \nu)^{1 / 2},
\end{array}
$$

equations (1)-(7) reduce to

$$
\begin{aligned}
m^{\prime \prime \prime}+2 m m^{\prime \prime}-\left(m^{\prime}\right)^{2}+G^{2}-\beta^{2} M^{2} & =0, \\
2\left(m G^{\prime}-m^{\prime} G\right)+G^{\prime \prime} & =0, \\
M^{\prime \prime}+2 \alpha m M^{\prime} & =0, \\
S^{\prime}+4 m m^{\prime}+2 m^{\prime \prime} & =0
\end{aligned}
$$

where $\alpha=\nu / \eta$ is the magnetic Prandil number and $\beta=\Omega / \omega$ is the ratio of the Alfven speed to the disk speed. Note that equation (12) decouples from the others, and $S(\zeta)$ is easily obtained once $m(\varsigma)$ is known.

It is assumed that the disk is porous and that either suction or injection occurs at the disk surface such that

$$
w(0)=-2 \sqrt{\nu \omega} A,
$$

so $A>0$ corresponds to suction and $A<0$ corresponds to injection.

The boundary conditions for equations (9)-(12) are,

$$
\begin{gathered}
m(0)=A, \quad m^{\prime}(0)=0, \quad G(0)=1, \quad M(0)=1, \\
m^{\prime}(\varsigma) \rightarrow 0, \quad G(\varsigma) \rightarrow 0, \quad M(\varsigma) \rightarrow 0, \quad s(\varsigma) \rightarrow 0 \quad \text { as } \varsigma \rightarrow \infty
\end{gathered}
$$

\section{NUMERICAL METHOD.}

The computation of $S(\zeta)$ is straightforward, and is not discussed here. Following the format in Heruska [11], define

$$
X=\left(\begin{array}{c}
m^{\prime \prime}(0) \\
G^{\prime}(0) \\
M^{\prime}(0)
\end{array}\right)
$$

Let $m(\varsigma ; X), G(\varsigma ; X), M(\zeta ; X)$ be the solution of the initial value problem given by equations (9)(11) with initial conditions (13) and (15). The original two-point boundary value problem given by equations (9)-(11) and (13) is numerically equivalent to solving the nonlinear system of equations

$$
F(X)=\left(\begin{array}{c}
m^{\prime}(r ; X) \\
G(r ; X) \\
M(r ; X)
\end{array}\right)=0
$$

where $T$ is chosen large enough such that

$$
\left|m^{\prime}(\zeta)-m^{\prime}(\tau)\right|+|G(\varsigma)-G(\tau)|+|M(s)-M(\tau)|<\epsilon
$$


for $\tau<\zeta<\infty$ and a given $\epsilon>0$. Equation (16) is derived from the boundary conditions (14). Algorithms for solving nonlinear systems like (16) typically require partial derivatives such as $\partial m^{\prime} / \partial X_{k}$. We can write the functions needed as

$$
Y=\left(m, m^{\prime}, m^{\prime \prime}, G, G^{\prime}, M, M^{\prime}, \frac{\partial m}{\partial X_{k}}, \frac{\partial m^{\prime}}{\partial X_{k}}, \frac{\partial m^{\prime \prime}}{\partial X_{k}}, \frac{\partial G}{\partial X_{k}}, \frac{\partial G^{\prime}}{\partial X_{k}}, \frac{\partial M}{\partial X_{k}}, \frac{\partial M^{\prime}}{\partial X_{k}}\right)
$$

for $k=1,2,3$, where $m, G$ and $M$ are functions of $\varsigma$. Now $m^{\prime}(\varsigma), G(\varsigma), M(\varsigma)$ and their partial derivatives can be calculated from the first order system

$$
\begin{aligned}
Y_{1}^{\prime} & =Y_{2} \\
Y_{2}^{\prime} & =Y_{3} \\
Y_{3}^{\prime} & =\beta^{2} Y_{6}^{2}+Y_{2}^{2}-Y_{4}^{2}-2 Y_{1} Y_{3} \\
Y_{4}^{\prime} & =Y_{5} \\
Y_{8}^{\prime} & =-2\left(Y_{1} Y_{5}-Y_{2} Y_{4}\right) \\
Y_{6}^{\prime} & =Y_{7} \\
Y_{7}^{\prime} & =-2 \alpha Y_{1} Y_{7} \\
Y_{8}^{\prime} & =Y_{9} \\
Y_{9}^{\prime} & =Y_{10} \\
Y_{10}^{\prime} & =2\left(\beta^{2} Y_{6} Y_{13}-Y_{4} Y_{11}-Y_{3} Y_{8}-Y_{1} Y_{10}+Y_{2} Y_{9}\right) \\
Y_{11}^{\prime} & =Y_{12} \\
Y_{12}^{\prime} & =2\left(Y_{9} Y_{4}+Y_{2} Y_{11}-Y_{1} Y_{12}-Y_{8} Y_{5}\right) \\
Y_{13}^{\prime} & =Y_{14} \\
Y_{14} & =-2 \alpha\left(Y_{8} Y_{7}+Y_{1} Y_{14}\right) \\
Y(0) & =\left(A, 0, X_{1}, 1, X_{2}, 1, X_{3}, 0,0, \delta_{1 k}, 0, \delta_{2 k}, 0, \delta_{3 k}\right)
\end{aligned}
$$

where $\delta_{i k}$ is the Kronecker delta. By solving this system three times, for $k=1,2,3$, the Jacobian matrix $D F(X)$ of $F(X)$ can be calculated.

Two methods were utilized to solve this problem, a quasi-Newton least change secant update method and a globally convergent homotopy method. The quasi-Newton code used was HYBRJ from the MINPACK subroutine package from Argonne National Laboratory [12]. These quasi-Newton routines are robust and usually quite efficient. However, they fail at times by converging to local minima of $F(X)^{t} F(X)$ which are not solutions of $F(X)=0$.

The other method, a globally convergent homotopy method from the subroutine package HOMPACK developed by Watson [17], does not suffer from the convergence problems of the quasi-Newton method. However, this method requires considerably more computation time. Details about the homotopy algorithm and some of its applications can be found in [13], [14], [15] and [16].

The computational strategy here was to try to solve (16) first using the relatively inexpensive quasi-Newton method. If that failed, then the expensive, but guaranteed convergent, homotopy algorithm was used. 
As previously mentioned, these quasi-Newton and homotopy methods use some partial derivatives with respect to the initial conditions. For some values of $A, \alpha$ and $\beta$, these partials increase drastically as $\tau$ increases. This results in numerical instability in cases where $A<-1$ (i.e., large injection) and $\alpha>10$. Also, cases of large injection and large $\beta$ cause $M(\varsigma)$ to approach zero very slowly. This requires $\tau$ to be very large which also causes numerical instability. Such instabilities are inherent with shooting, on which the definition of $F(X)$ is based. Other ways of discretizing the two-point boundary value problem (9)-(14) such as finite differences, collocation, and finite elements will be considered in future work.

\section{DISCUSSION.}

The rotating disk acts as a centrifugal fan. The radial flow is balanced by an induced axial flow towards the rotating disk. When suction is applied at the disk surface, the radial flow decreases, the axial flow at infinity increases towards the disk; and the boundary layer thickness decreases. The opposite effect is observed when the fluid is injected at the disk surface; the radial velocity increases, the axial velocity at infinity decreases and the boundary layer thickens. With an impermeable rotating disk, an increase in $\beta$ decreases the axial velocity at infinity and increases the boundary layer thickness. As $\alpha$ increases, the boundary layer thicknesses of the flow and magnetic feld decrease.

Figure 1 shows the effect of $A$ on $m^{\prime}, G, m$ and $M$ for $\alpha=.5$ and $\beta=.2$. The case $A=0$ corresponds to the impermeable disk [10] in all the figures. The effect of $A$ on axial velocity is shown in Figure 1a. As suction $(A>0)$ increases, the axial velocity becomes almost constant and maintains the suction value throughout the boundary layer. When fiuid is injected at the disk surface $(A<0)$, the incoming axial flow is retarded by the injected fluid. The greater the injection velocity, the more the inflow is opposed. This results in decreasing the axial velocity at infinity and moving the crossover point away from the disk. The radial velocity, $m^{\prime}$, is shown in Figure $1 \mathrm{~b}$. The radial velocity increases monotonically from zero velocity at the disk surface to a maximum near the disk and then decreases monotonically to zero at the edge of the boundary layer. As the injection velocity increases, the maximum for radial velocity increases and moves away from the disk. Increased suction velocity decreases the maximum and moves it closer to the disk. The radial velocity becomes almost zero for large suction values causing the flow to be two dimensional. Figure 1c shows the variation in tangential velocity, $G$, due to changes in $A$. The boundary layer thickness decreases with increased suction and increases with increased injection velocity. The thickness of the boundary layer for the magnetic field $M$ also increases with injection and decreases with suction (Figure 1d).

Figure 2 shows the effect of $\alpha$ on the flows with a fxed value of $\beta$ and a fixed injection relocity $(A=-.5)$. The effect of $\alpha$ on axial velocity is shown in Figure 2a. An increase in $\alpha$ causes only a small increase in $m$ near the disk. A larger increase occurs at infinity. However, this increase diminishes as $\alpha$ gets large; there is almost no change in $m$ between $\alpha=5$ and $\alpha=10$. Similarly, the effect of an increase in $\alpha$ on the radial velocity (Figure $2 \mathrm{~b}$ ) is a small and diminishing increase. The effect on tangential velocity (Figure $2 c$ ) is small also, but decreases the tangential velocity. The effect of $\alpha$ on the magnetic field $M$ is shown in Figure 2d. For large values of $\alpha$, the magnetic field maintains the same strength as at the disk surface for a small distance from the disk and then falls to its edge strength in a very short distance. A higher value of injection $(A=-1)$ gives similar effects of $\alpha$ on $m, m^{\prime}$ and $G$ (not shown), but has a more pronounced effect on $M$ (Figure 2e). The 
same effect of $\alpha$ on $m, m^{\prime}$, and $G$ are observed when there is suction $(A=.5)$ at the disk surface. The only relevant difference is that $m^{\prime}$ peaks at a much lower level. The effect of increasing $\alpha$ on $M$ in the presence of suction is to not maintain the initial strength for a short distance from the disk surface, but to fall to the edge strength quickly (Figure 2f).

Figure 3 shows the effect of $\beta$ on the flow fields when fivid is injected at the disk surface $(A=-.5)$ and $\alpha=1$. The axial velocity (Figure $3 \mathrm{a}$ ) is decreased as $\beta$ increases. The effect is more pronounced at infinity. As $\beta$ gets large, an increase in $\beta$ causes larger decreases in $m$ at infinity. The crossover point from negative to positive flow moves away from the disk as $\beta$ increases. The radial velocity (Figure 3b) decreases as $\beta$ increases. Again, this change increases as $\beta$ increases. But, tangential velocity (Figure 3c) and the magnetic field strength increase as $\beta$ increases (Figure 3d).

Figure 4 shows the effect of $\beta$ on the flow fields when suction $(A=1)$ is applied at the disk surface and $\alpha=1$. As $\beta$ gets large, the axial flow (Figure 4a) towards the disk decreases and becomes a constant throughout the boundary layer when $\beta=1$. The radial velocity (Figure $4 \mathrm{~b}$ ) decreases as $\beta$ increases and disappears at $\beta=1$. The effect of $\beta$ on the tangential velocity and the magnetic field is not very pronounced, so is not shown.

From Table 1, we can see that changes in $A$ have a more pronounced effect on $G^{\prime}(0)$ than do changes in $\alpha$ and $\beta$. Changes in $\alpha$ have a very small effect on $G^{\prime}(0)$. As $A$ gets smaller (which decreases $\left|G^{\prime}(0)\right|$ ), the effect of $A$ decreases. However, as $\beta$ gets larger (which also decreases $\left|G^{\prime}(0)\right|$ ), there is an increasing effect on $G^{\prime}(0)$.

From Table 1, critical values (where $G^{\prime}(0)=0$ ) of $A, \alpha$ and $\beta$ would occur for small $A$ and large $\beta$. It is apparent from the $M^{\prime}(0)$ column that $M^{\prime}(0)$ approaches zero faster than $G^{\prime}(0)$ does. Thus shooting needs to use a large $\tau$ for $G$ and $M$ to converge to zero. As previously mentioned, this results in numerical instability, and considerable computational diffeulty was encountered attempting to find critical values. To find values for $A, \alpha$ and $\beta$ where $G^{\prime}(0)=0$, an expensive and involved multiple shooting technique [18] was employed. Using this technique, values for $G^{\prime}(0)$ close to zero were obtained varying $\beta$ and keeping $A=.1$ and $\alpha=1.0$. Using three data points close to zero, quadratic extrapolation to the limit yielded $\beta=1.1072$.

Table 2 shows excellent agreement of our results with the approximate analytic solution obtained by $\mathrm{Pao}[10]$ for $A=0$. The maximum percent difference in Table 2 is $0.197 \%$ for $m^{\prime \prime}(0), 0.174 \%$ for $G^{\prime}(0), 0.391 \%$ for $M^{\prime}(0)$, and $2.016 \%$ for $2 m(\infty)$, the latter occuring at $\alpha=1.0, \beta=.70$.

\section{ASYMPTOTIC COMPARISONS.}

Applying standard perturbation techniques to equations (9)-(11) for large $A$ and small $\alpha$ and $\beta$ we get

$$
\begin{aligned}
G^{\prime}(0) & \approx \hat{G}^{\prime}(0)=-2 A, \\
M(0) & \approx \hat{M}^{\prime}(0)=-2 A \alpha, \\
m^{\prime \prime}(0) \approx \hat{m}^{\prime \prime}(0) & =\frac{\alpha-\beta^{2}}{4 A \alpha} .
\end{aligned}
$$

From Table 3, we see that the asymptotic formula values compare favorably with obtained results for $A \geq 1$. Also, changes in $\alpha$ do not affect the goodness of the asymptotic formulas within the range studied. Furthermore, there is good agreement for $\beta$ values less than 1.0. 


\begin{tabular}{|c|c|c|c|c|c|c|}
$A$ & $\alpha$ & $\beta$ & $2 m(\infty)$ & $m^{\prime \prime}(0)$ & $-M^{\prime}(0)$ & $-G^{\prime}(0)$ \\
\hline 4.00 & .5 & .2 & 8.0008 & .05749 & 4.00023 & 8.00057 \\
2.00 & .5 & .2 & 4.0066 & .11479 & 2.00180 & 4.00456 \\
1.00 & .5 & .2 & 2.0490 & .22387 & 1.01351 & 2.03422 \\
.50 & .5 & .2 & 1.2301 & .36553 & .56466 & 1.16202 \\
0.00 & .5 & .2 & .8223 & .48806 & .25234 & .60054 \\
-0.05 & .5 & .2 & .7999 & .49210 & .22968 & .56088 \\
-1.00 & .5 & .2 & .5111 & .38300 & .01678 & .13083 \\
\hline-0.50 & .5 & .2 & .6560 & .46997 & .08367 & .29220 \\
-0.50 & 1.0 & .2 & .7249 & .46982 & .06460 & .29280 \\
-0.50 & 5.0 & .2 & .7395 & .46826 & .00072 & .29178 \\
-0.50 & 10.0 & .2 & .7392 & .46799 & .00000 & .29138 \\
\hline-1.00 & .5 & .2 & .5111 & .38300 & .01678 & .13083 \\
-1.00 & 1.0 & .2 & .6441 & .38278 & .00334 & .13070 \\
-1.00 & 5.0 & .2 & .6642 & .38267 & .00000 & .13055 \\
\hline .50 & .5 & .2 & 1.2301 & .36553 & .56466 & 1.16202 \\
.50 & 1.0 & .2 & 1.2530 & .37531 & 1.08979 & 1.17023 \\
.50 & 5.0 & .2 & 1.2602 & .38587 & 5.07951 & 1.17484 \\
.50 & 10.0 & .2 & 1.2605 & .38764 & 10.05441 & 1.17511 \\
\hline-0.50 & 1.0 & 0.0 & .7607 & .48948 & .06982 & .30217 \\
-0.50 & 1.0 & .1 & .7524 & .48459 & .06855 & .29988 \\
-0.50 & 1.0 & .2 & .7249 & .46982 & .06461 & .29280 \\
-0.50 & 1.0 & .3 & .6680 & .44495 & .05391 & .28026 \\
-0.50 & 1.0 & .4 & .5212 & .40954 & .04441 & .26031 \\
\hline 1.00 & 1.0 & 0.0 & 2.0578 & .24242 & 2.01941 & 2.03853 \\
1.00 & 1.0 & .2 & 2.0556 & .23290 & 2.01867 & 2.03707 \\
1.00 & 1.0 & .6 & 2.0378 & .15625 & 2.01266 & 2.02519 \\
1.00 & 1.0 & 1.0 & 2.0000 & .00000 & 2.00000 & 2.00000 \\
& & & & &
\end{tabular}

Table 1. Effect of $A, \alpha$, and $\beta$ on $m(\infty), m^{\prime \prime}(0), M^{\prime}(0)$ and $G^{\prime}(0)$. 


\begin{tabular}{|r|r|r|r|r|r|r|r|r|r|}
\multicolumn{1}{|c|}{$\alpha$} & \multicolumn{1}{|c|}{$\beta$} & $\hat{m}^{\prime \prime}(0)$ & $m^{\prime \prime}(0)$ & $-\hat{G}^{\prime}(0)$ & $-G^{\prime}(0)$ & $-\hat{M}^{\prime}(0)$ & $-M^{\prime}(0)$ & $2 \hat{m}(\infty)$ & $2 m(\infty)$ \\
\hline .5 & 0.00 & .51024 & .51023 & .61592 & .61592 & .26227 & .26229 & .884 & .884 \\
.5 & .05 & .50886 & .50885 & .61501 & .61501 & .26170 & .26172 & .882 & .881 \\
.5 & .10 & .50470 & .50471 & .61223 & .61223 & .25997 & .25997 & .871 & .870 \\
.5 & .15 & .49778 & .49779 & .60749 & .60748 & .25696 & .25692 & .852 & .852 \\
.5 & .20 & .48806 & .48806 & .60054 & .60054 & .25236 & .25234 & .823 & .822 \\
.5 & .25 & .47550 & .47550 & .59102 & .59100 & .24573 & .24566 & .778 & .777 \\
\hline 1.0 & 0.00 & .51024 & .51023 & .61592 & .61592 & .39625 & .39625 & .884 & .884 \\
1.0 & .10 & .50558 & .50558 & .61362 & .61363 & .39469 & .39469 & .880 & .880 \\
1.0 & .20 & .49154 & .49154 & .60657 & .60657 & .38991 & .38991 & .867 & .867 \\
1.0 & .30 & .46786 & .46786 & .59423 & .59423 & .38152 & .38512 & .844 & .844 \\
1.0 & .40 & .43406 & .43405 & .57552 & .57552 & .36872 & .36874 & .809 & .809 \\
1.0 & .50 & .38936 & .38936 & .54836 & .54834 & .34998 & .34988 & .755 & .755 \\
1.0 & .60 & .33266 & .33265 & .50822 & .50816 & .32186 & .32175 & .669 & .669 \\
1.0 & .70 & .26296 & .26289 & .44319 & .44242 & .27451 & .27344 & .506 & .496 \\
\hline 10.0 & 0.00 & .51024 & .51023 & .61592 & .61592 & 1.13399 & 1.13412 & .884 & .884 \\
10.0 & .20 & .50058 & .50057 & .61378 & .61378 & 1.12916 & 1.12915 & .883 & .883 \\
10.0 & .40 & .47120 & .47119 & .60717 & .60717 & 1.11380 & 1.11379 & .878 & .878 \\
10.0 & .60 & .42078 & .42078 & .59540 & .59540 & 1.08638 & 1.08638 & .870 & .870 \\
10.0 & .80 & .34650 & .34650 & .57695 & .57695 & 1.04322 & 1.04322 & .857 & .857 \\
10.0 & 1.00 & .24202 & .24201 & .54816 & .54816 & .97545 & .97545 & .838 & .838 \\
10.0 & 1.20 & .08714 & .08713 & .49600 & .49600 & .85155 & .85155 & .805 & .805 \\
10.0 & 1.30 &. .07124 &. .07110 & .41399 & .41412 & .65578 & .65606 & .757 & .759 \\
& & & & & & & &
\end{tabular}

Table 2. Comparison of current results with those of Pao (hatted).

\begin{tabular}{|r|r|r|r|r|r|r|r|r|r|r|r|}
\multicolumn{1}{|c|}{$\alpha$} & \multicolumn{1}{|c|}{$\alpha$} & \multicolumn{1}{|c|}{$\beta$} & $m^{\prime \prime}(0)$ & $\hat{m}^{\prime \prime}(0)$ & $\%$ diff & $-G^{\prime}(0)$ & $-\hat{G}^{\prime}(0)$ & $\%$ diff & $-\boldsymbol{M}^{\prime}(0)$ & $-\hat{M}^{\prime}(0)$ & $\%$ diff \\
\hline 4.0 & 0.5 & 0.2 & .05749 & .0575 & .017 & 8.00057 & 8 & .007 & 4.00023 & 4.0 & .006 \\
2.0 & 0.5 & 0.2 & .11479 & .1150 & .183 & 4.00456 & 4 & .114 & 2.00180 & 2.0 & .090 \\
1.0 & 0.5 & 0.2 & .22387 & .2300 & 2.738 & 2.03422 & 2 & 1.682 & 1.01351 & 1.0 & 1.333 \\
0.5 & 0.5 & 0.2 & .36553 & .4600 & 25.845 & 1.62020 & 1 & 13.652 & .56466 & .5 & 11.451 \\
\hline 2.0 & 0.5 & 0.2 & .11479 & .1150 & .183 & 4.00456 & 4 & .114 & 2.00180 & 2.0 & .090 \\
2.0 & 1.0 & 0.2 & .11975 & .1200 & .209 & 4.00497 & 4 & .124 & 4.00249 & 4.0 & .062 \\
2.0 & 5.0 & 0.2 & .12374 & .1240 & .210 & 4.00517 & 4 & .129 & 20.00185 & 20.0 & .009 \\
2.0 & 10.0 & 0.2 & .12424 & .1245 & .211 & 4.00518 & 4 & .129 & 40.00118 & 40.0 & .003 \\
\hline 2.0 & 0.5 & 0.2 & .11479 & .1150 & .183 & 4.00456 & 4 & .114 & 2.00180 & 2.0 & .090 \\
2.0 & 0.5 & 0.4 & .08491 & .0850 & .104 & 4.00270 & 4 & .067 & 2.00097 & 2.0 & .048 \\
2.0 & 0.5 & 0.6 & .03499 & .0350 & .033 & 3.99958 & 4 & .011 & 1.99958 & 2.0 & .021 \\
2.0 & 0.5 & 0.8 & .03517 & .0350 & .487 & 3.99517 & 4 & .121 & 1.99762 & 2.0 & .119 \\
2.0 & 0.5 & 1.0 & -.12585 &. .1250 & .672 & 3.98944 & 4 & .265 & 1.99507 & 2.0 & .247
\end{tabular}

Table 3 . Comparison of obtained ralues with asymptotic formula values (hatted). 


\section{REFERENCES.}

1. T. Von Karman, 'Uber Laminare und Turbulente Reibung', Zeit. angew. Math. Mech., 1, 233252 (1921).

2. W. G. Cochran, 'The Flow Due to a Rotating Disk', Proc. Camb. Phil. Soc, 30, 365-375 (1934).

3. J. T. Stuart, 'On the Effects of Uniform Suction on the Steady Flow due to a Rotating Disk', Quart. J. Mech. Appl. Math., 7, 446-457 (1954).

4. E. M. Sparrow and J. L. Gregg, 'Mass Transfer and Heat Transfer about a Rotating Disk', ASME J. Heat Trans., 81, 294-302 (1959).

5. H. K. Kuiken, 'The Effect of Normal Blowing on the Flow near a Rotating Disk of Infinite Extent', J. Fiuid Mech., 47, 789-798 (1971).

6. J. A. D. Ackroyd, 'On the Steady Flow Produced by a Rotating Disk with Either Surface Suction or Injection', J. Enga. Math., 12, 207-209 (1978).

7. E. M. Sparrow and R. D. Cess, 'Magnetohydrodynamic and Heat Transfer about a Rotating Disk', ASME J. Appl. Mech., 29, 181-187 (1962).

8. S. A. T. Rizvi, 'On the Steady Rotation of a Disc in Magnetohydrodynamics', Appl. Sci. Res., B10, 62-72 (1963).

9. G. S. Pande, 'On the Effects of Uniform High Suction on the Steady Hydromagnetic Flow due to a Rotating Disc', Appl. Sci. Res., B11, 205-212 (1964).

10. H. P. Pao, 'Magnetohydrodynamic flow over a Rotating. Disc', American Inst. Aero. Astro. Journal, 6, 1285-1291 (1968).

11. M. W. Heruska, 'Micropolar Flow past a Porous Stretching Sheet', M.S. Thesis, Virginia Polytechnic Institute and State University, Blacksburg, Va., Oct. 1984.

12. J. J. Moré, B. S. Garbow and K. E. Hillstrom, User Guide for MINPACK-1, ANL-80-74, Argonne National Laboratory, (1980).

13. L. T. Watson, 'A Globally Convergent Algorithm for Computing Fixed Points of $C^{2}$ Maps', Appl. Math. Comput., 5, 297-311 (1979).

14. L. T. Watson, 'Numerical Study of Porous Channel Flow in a Rotating System by a Homotopy Method', J. Comput. Appl. Math, 7, 21-26 (1981).

15. L. T. Watson and D. Fenner, 'Chow-Yorke Algorithm for Fixed Points or Zeros of $C^{2}$ Maps', ACM Trans. Math. Software, 6, 252-259 (1980).

16. L. T. Watson, 'Engineering applications of the Chow-Yorke Algorithm', Appl. Math. Comput., 9 (1981), 111-133.

17. L. T. Watson, S. C. Billups and A. P. Morgan, 'Hompack: A Suite of Codes for Globally Convergent Homotopy Algorithms', Tech. Report 85-34, Dept. of Industrial and Operations Engineering, University of Michigan, Ann Arbor, Michigan, 1985. 
18. H. B. Keller, Numerical Solution of Two-Point Boundary Value Problems, Society for Industrial and Applied Mathematics, Philadelphia, 1976.

Figure Captions.

Figure 1. Effect of $A$ on flow and magnetic fields with $\alpha=.5$ and $\beta=.2$ (a) Axial velocity, $A=4,2,1, .5,0,-.01,-1$ (top to bottom). (b) Radial velocity, $A=-1,-.05,0, .5,1,2,4$ (top to bottom). (c) Tangential velocity, $A=-1,-.05,0, .5,1,2,4$ (top to bottom). (d) Magnetic field, $A=-1,-.05,0, .5,1,2,4$ (top to bottom).

Figure 2. Effect of $\alpha$ on flow and magnetic fields with $\beta=.2$. (a) Axial velocity, $A=-.5$, $\alpha=10,1, .5$ (dotted, dashed, solid). (b) Radial velocity, $A=-.5, \alpha=10,1, .5$ (dotted, dashed, solid). (c) Tangential velocity. $A=-.5, \alpha=10, .5$ (dotted, dashed). (d) Magnetic field. $A=-.5, \alpha=10,5,1, .5$ (dotted, short dashed, long dashed, solid). (e) Magnetic field. $A=-1, \alpha=5,1, .5$ (dotted, dashed, solid). (f) Magnetic field. $A=.5$, $\alpha=10,5,1, .5$ (dotted, short dashed, long dashed, solid).

Figure 3. Effect of $\beta$ on flow and magnetic fields with $A=-.5, \alpha=1$ and $\beta=0, .1, .2, .3, .4$ (dotted, short dashed, long dashed, solid, dash-dot). (a) Axial velocity. (b) Radial velocity. (c) Tangential velocity. (d) Magnetic field.

Figure 4. Effect of $\beta$ on flow and magnetic fields with $A=1, \alpha=1$, and $\beta=0, .2, .6,1$ (dotted, dashed, solid, dash-dot). (a) Axial velocity. (b) Radial velocity. 


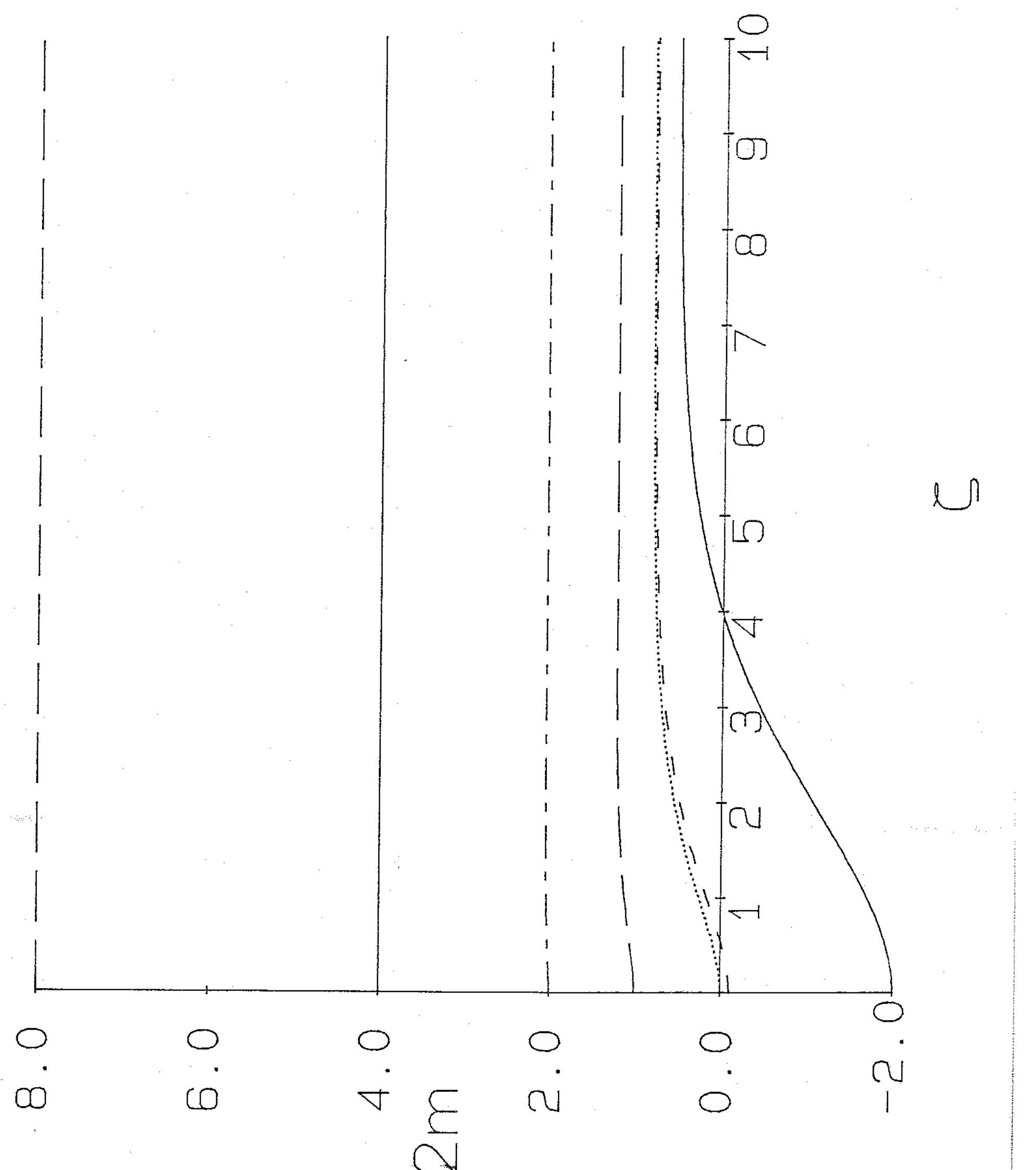


$\$$

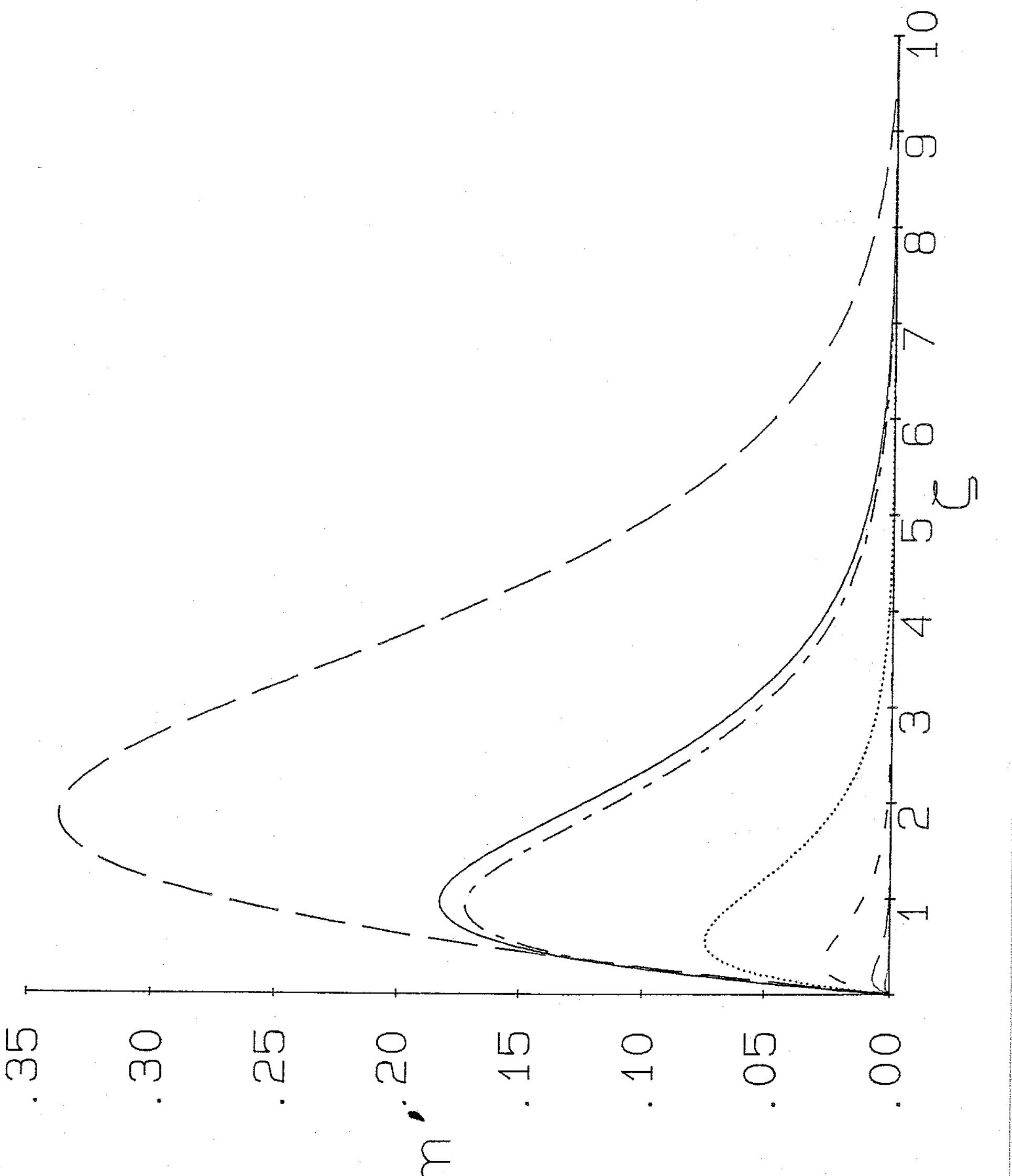


U

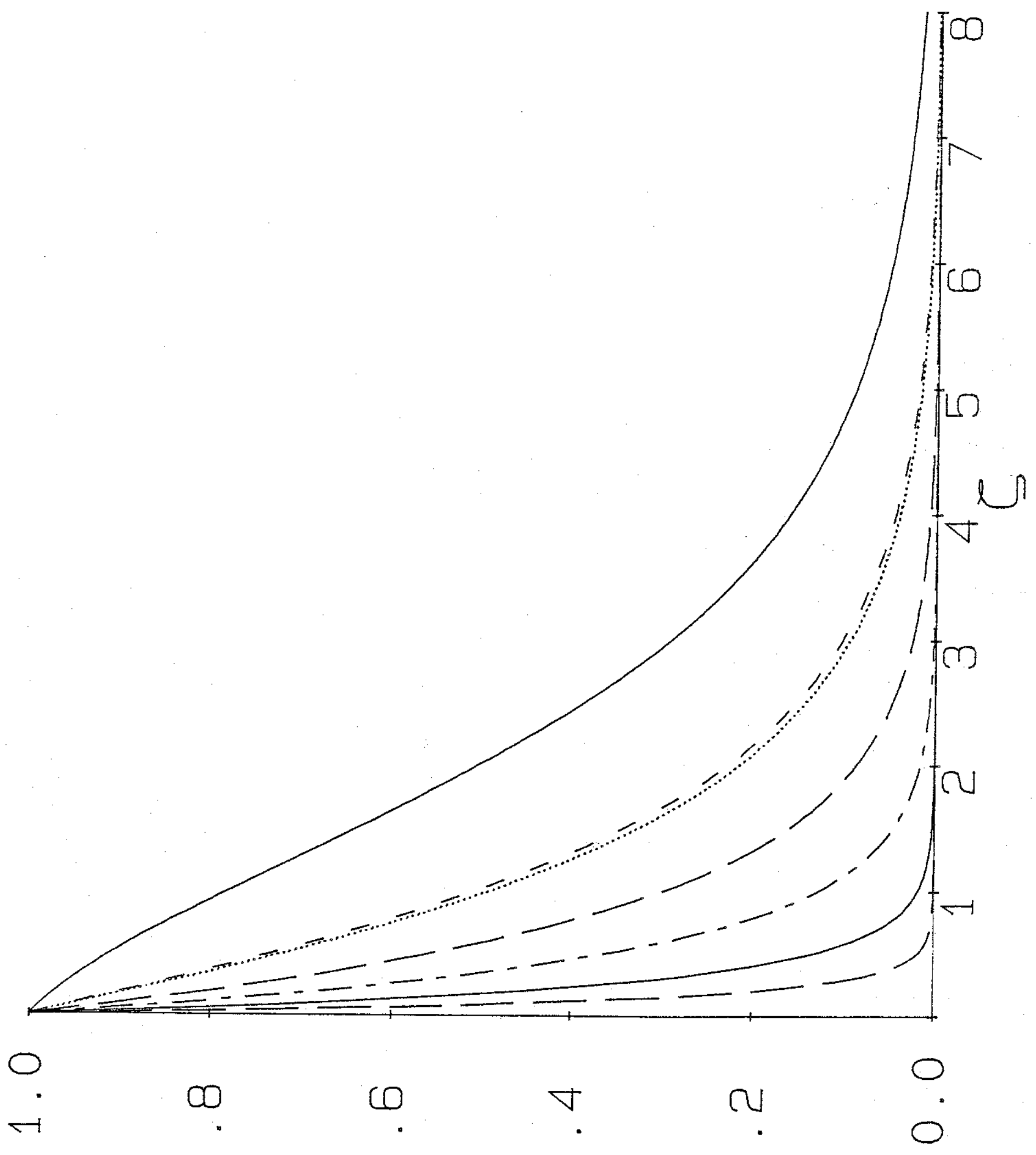

() 


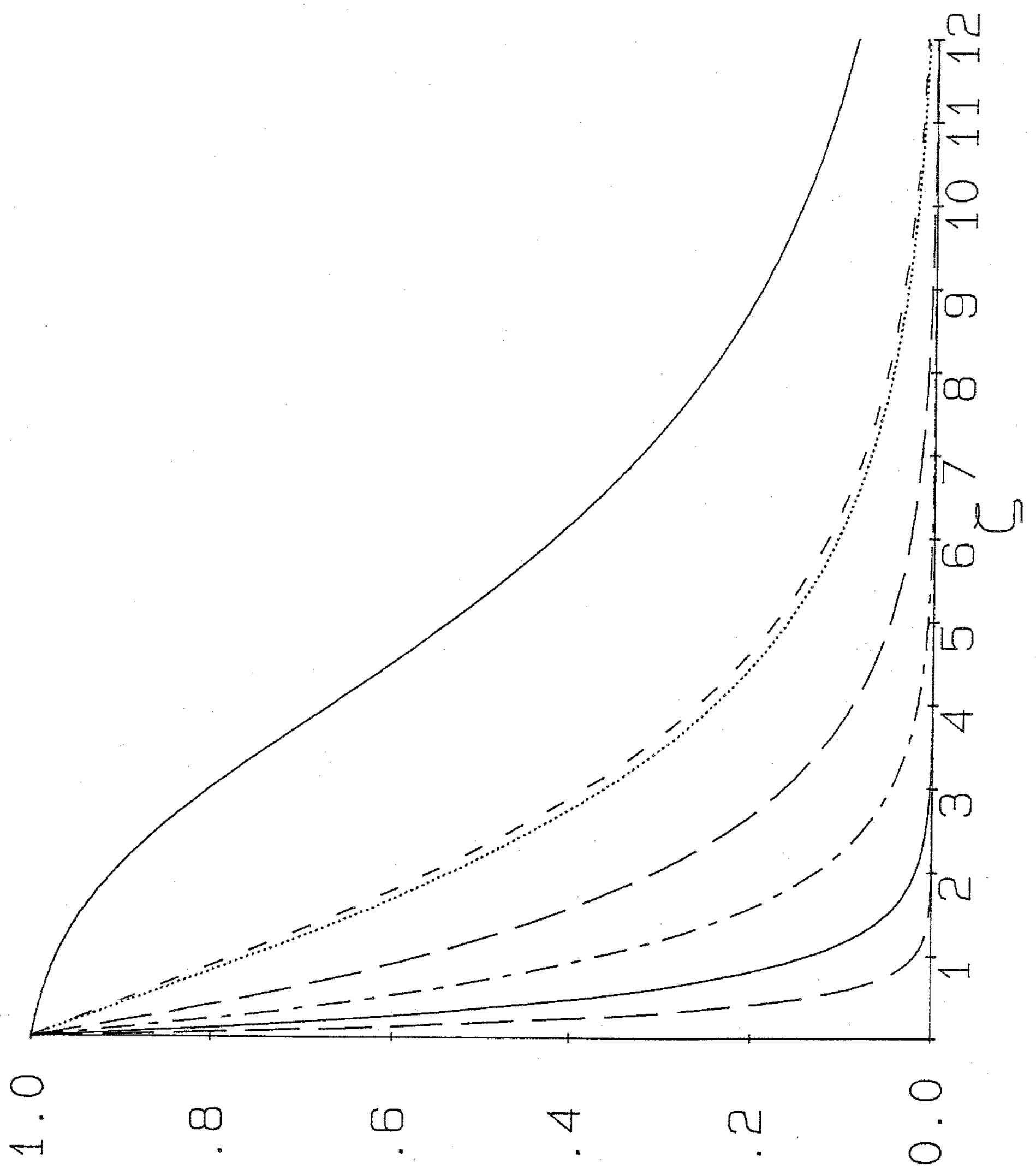

$\Sigma$ 


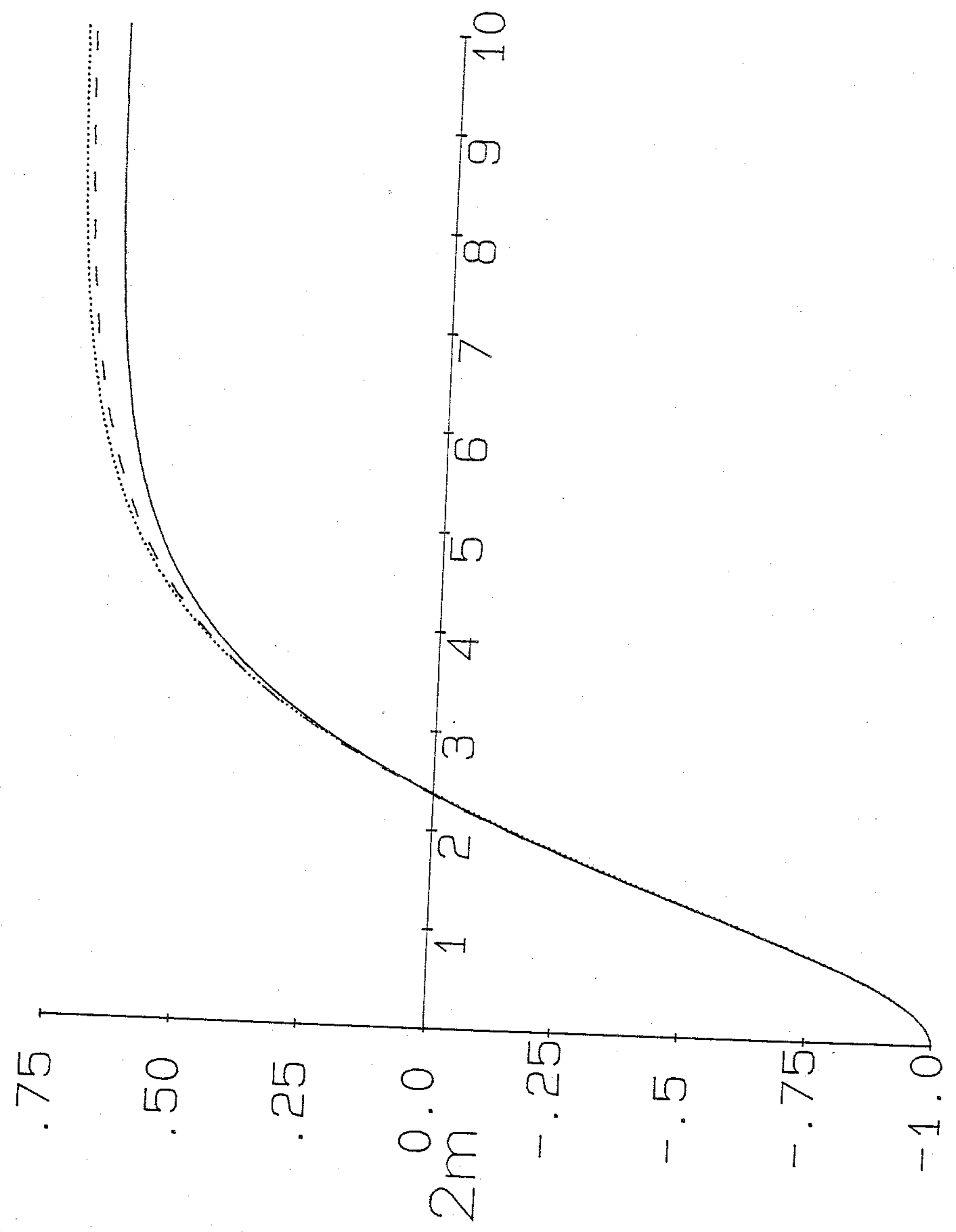


ชै

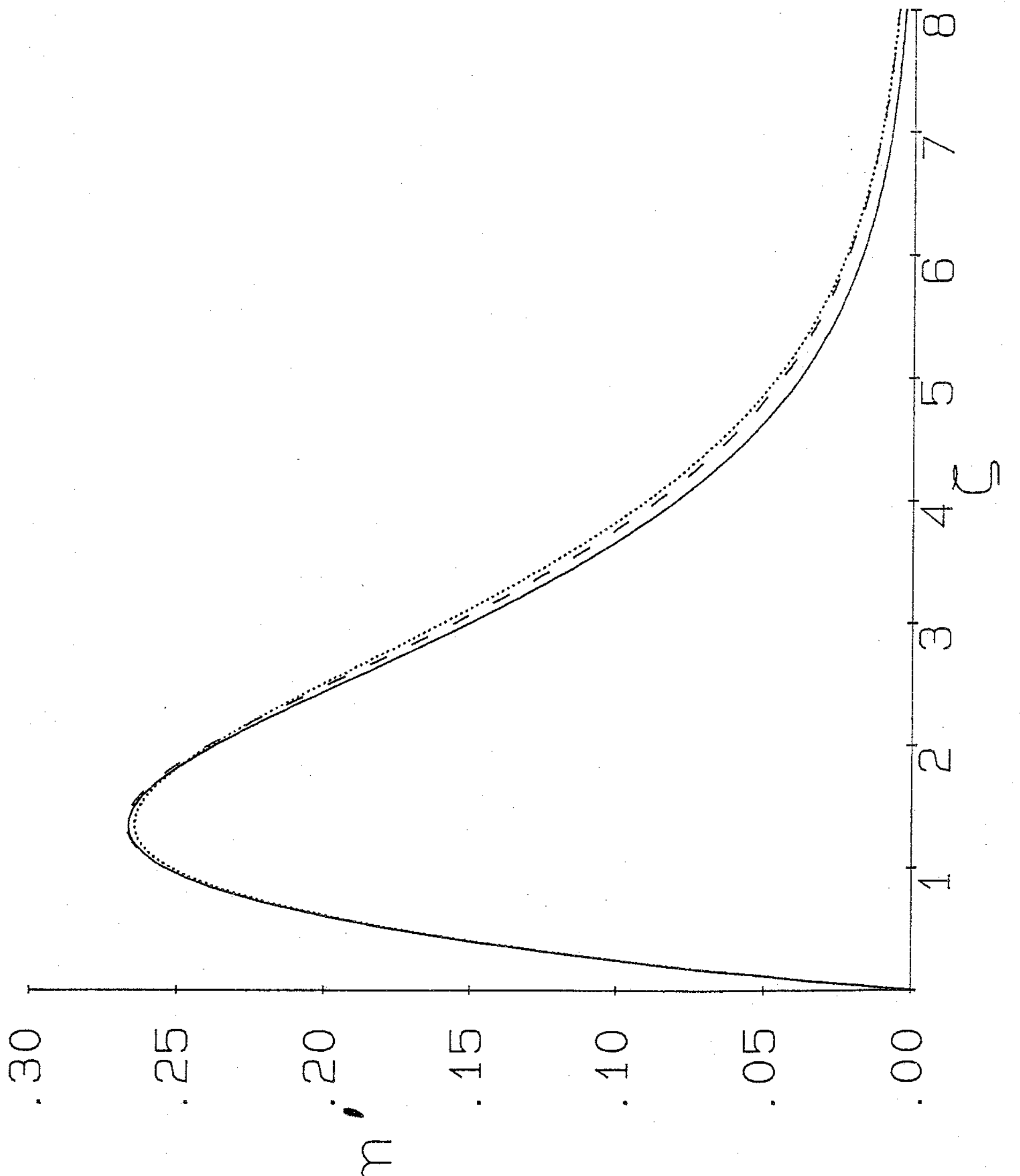


ช

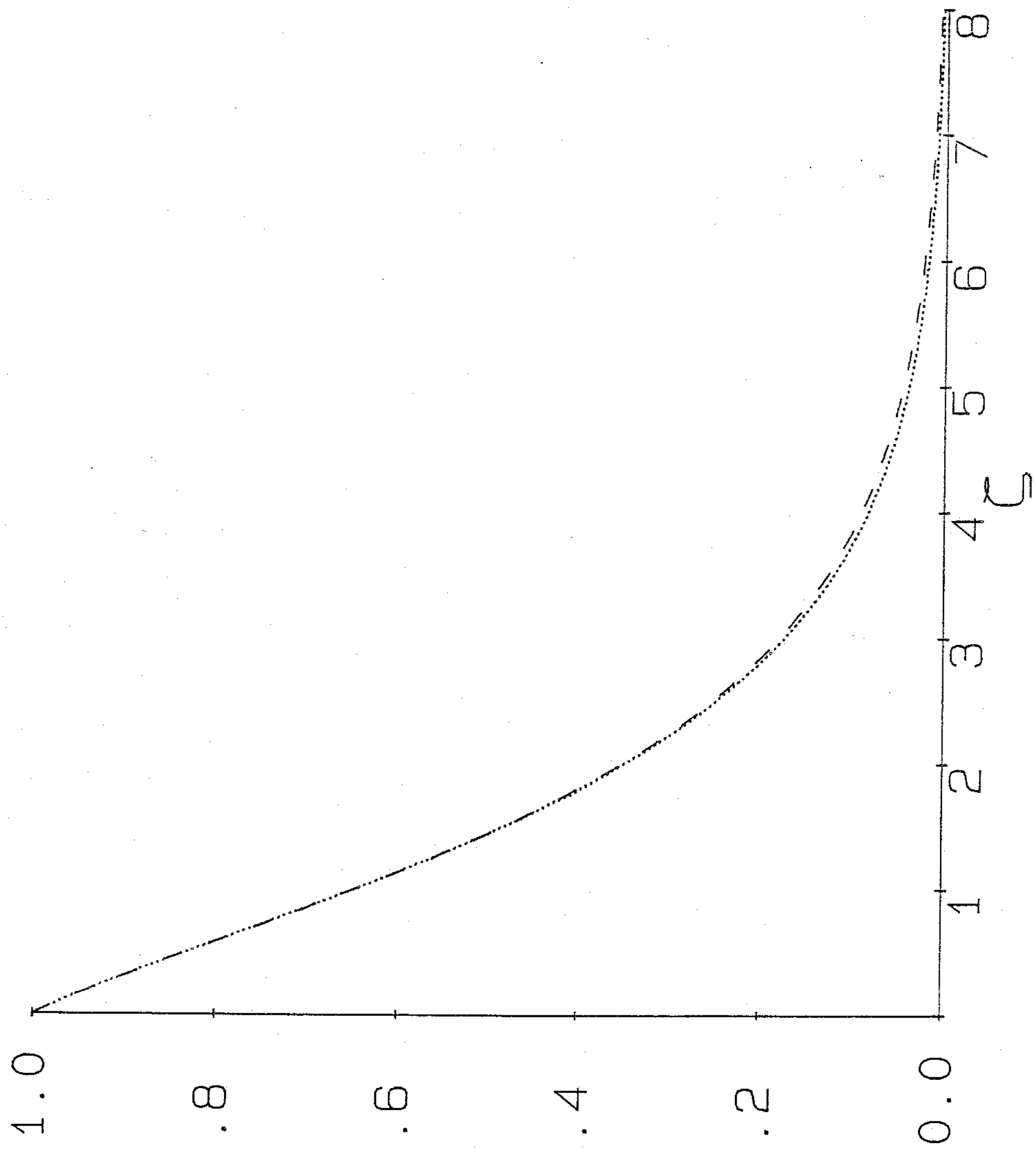

() 
$\frac{\sigma}{6}$

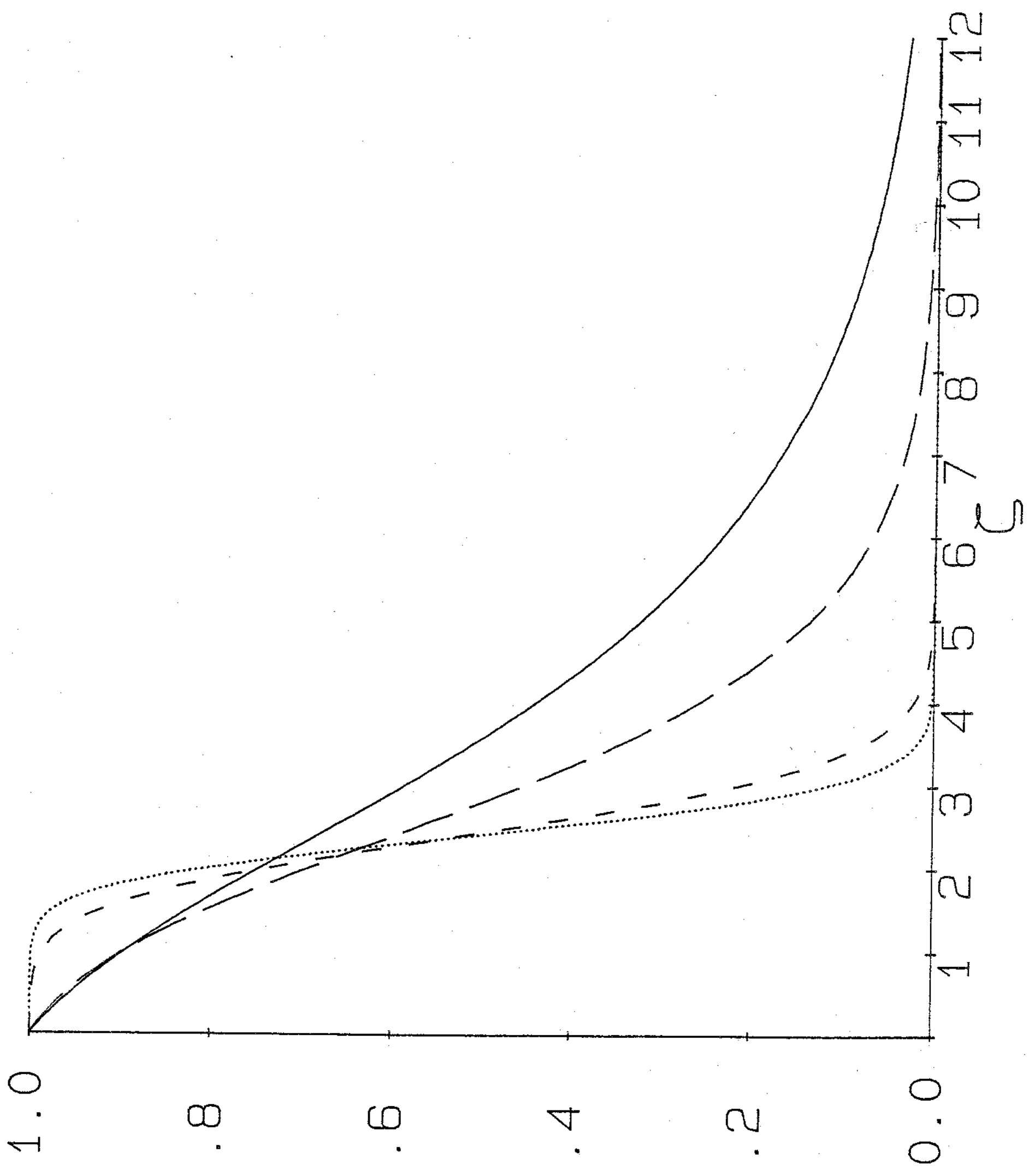




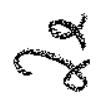

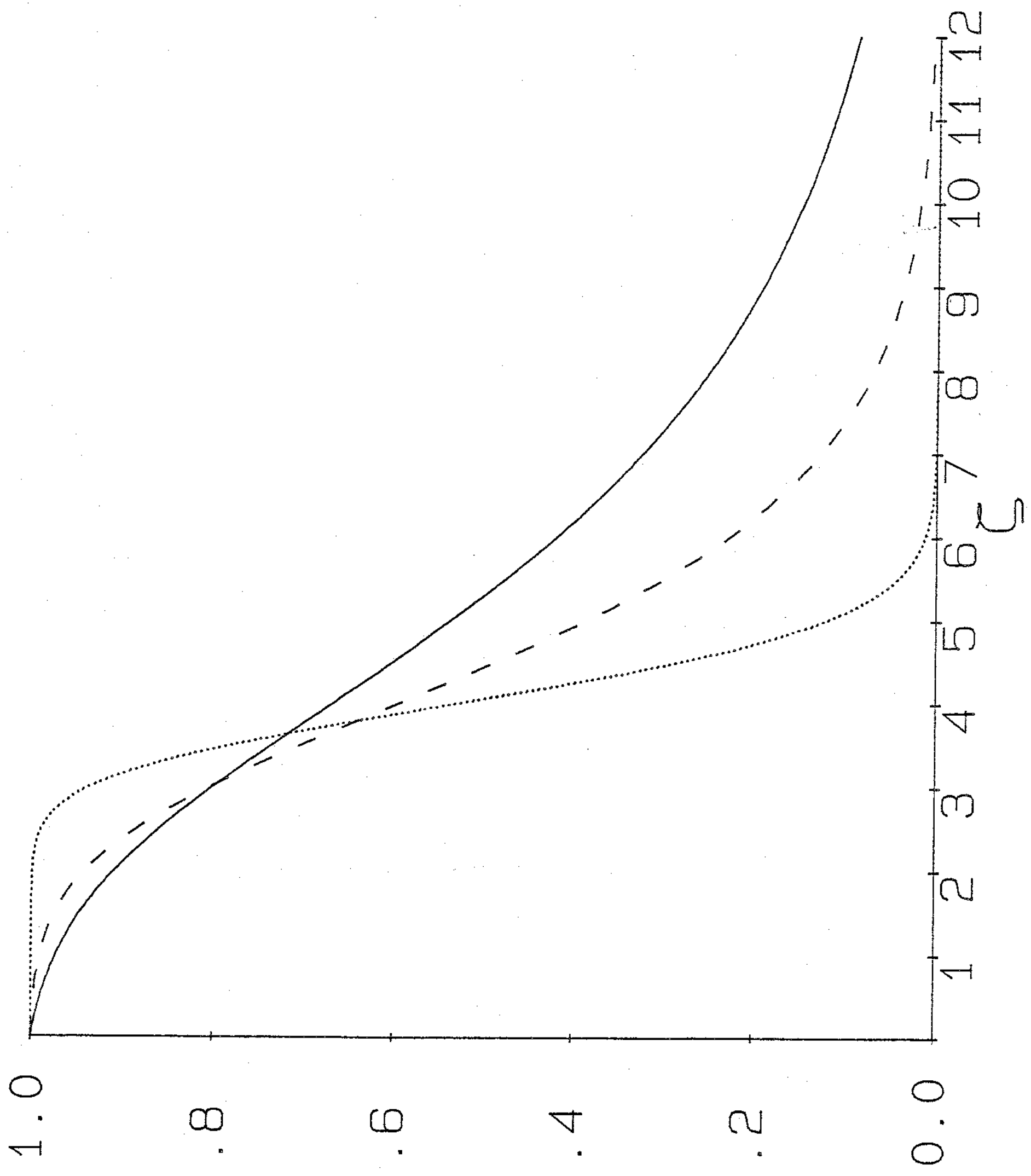


$-4$

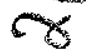

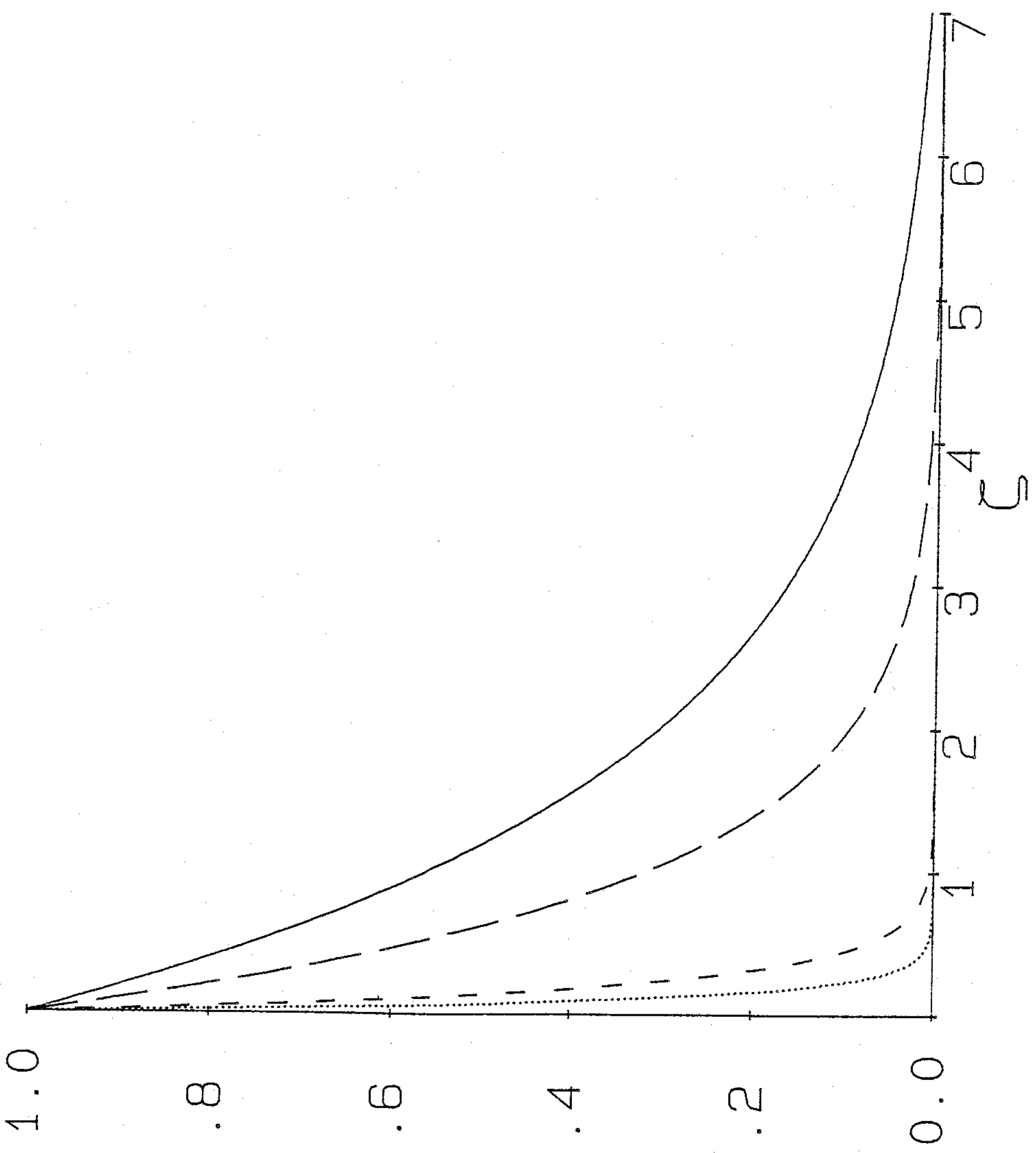

5 
๓

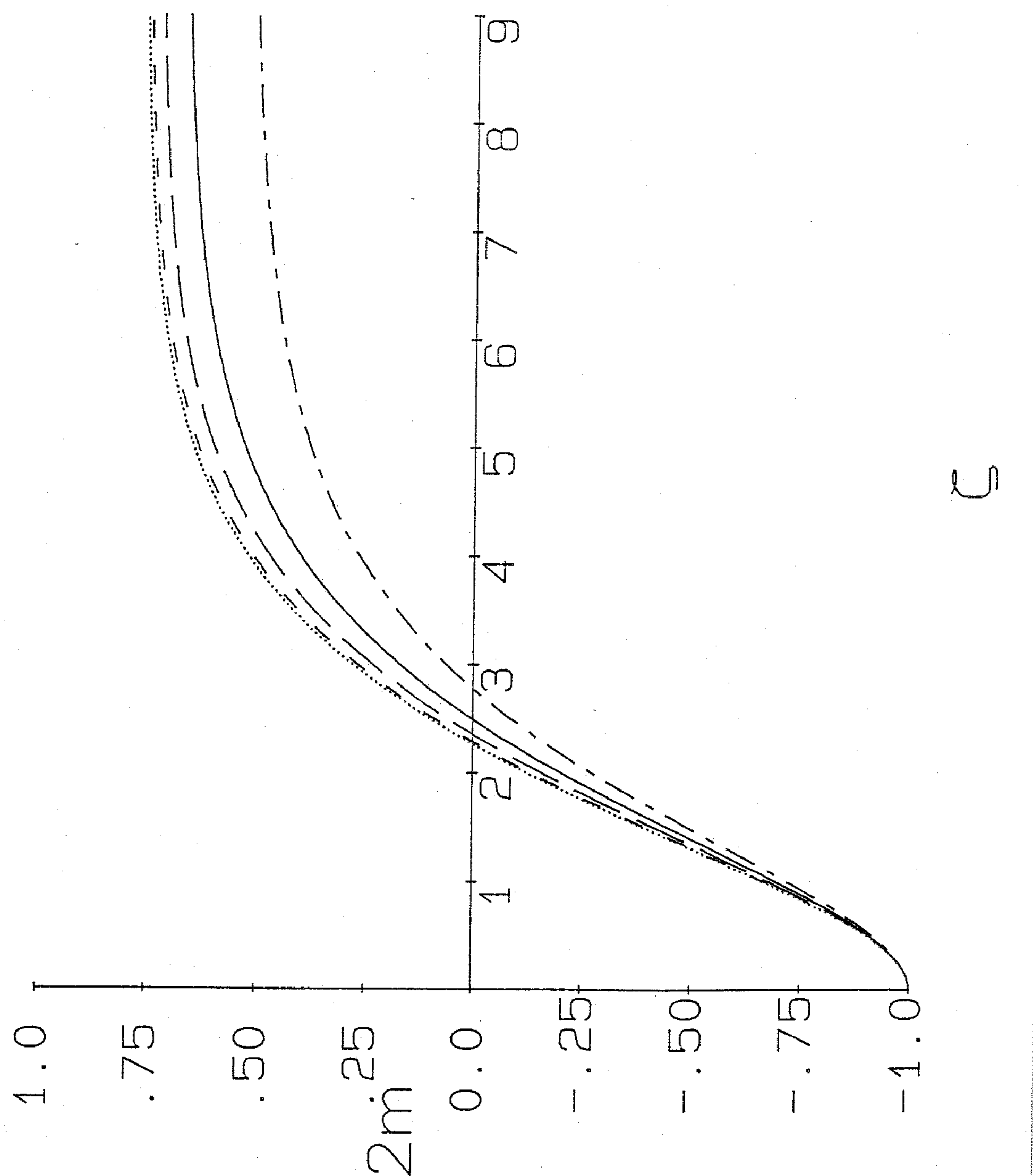


$m$

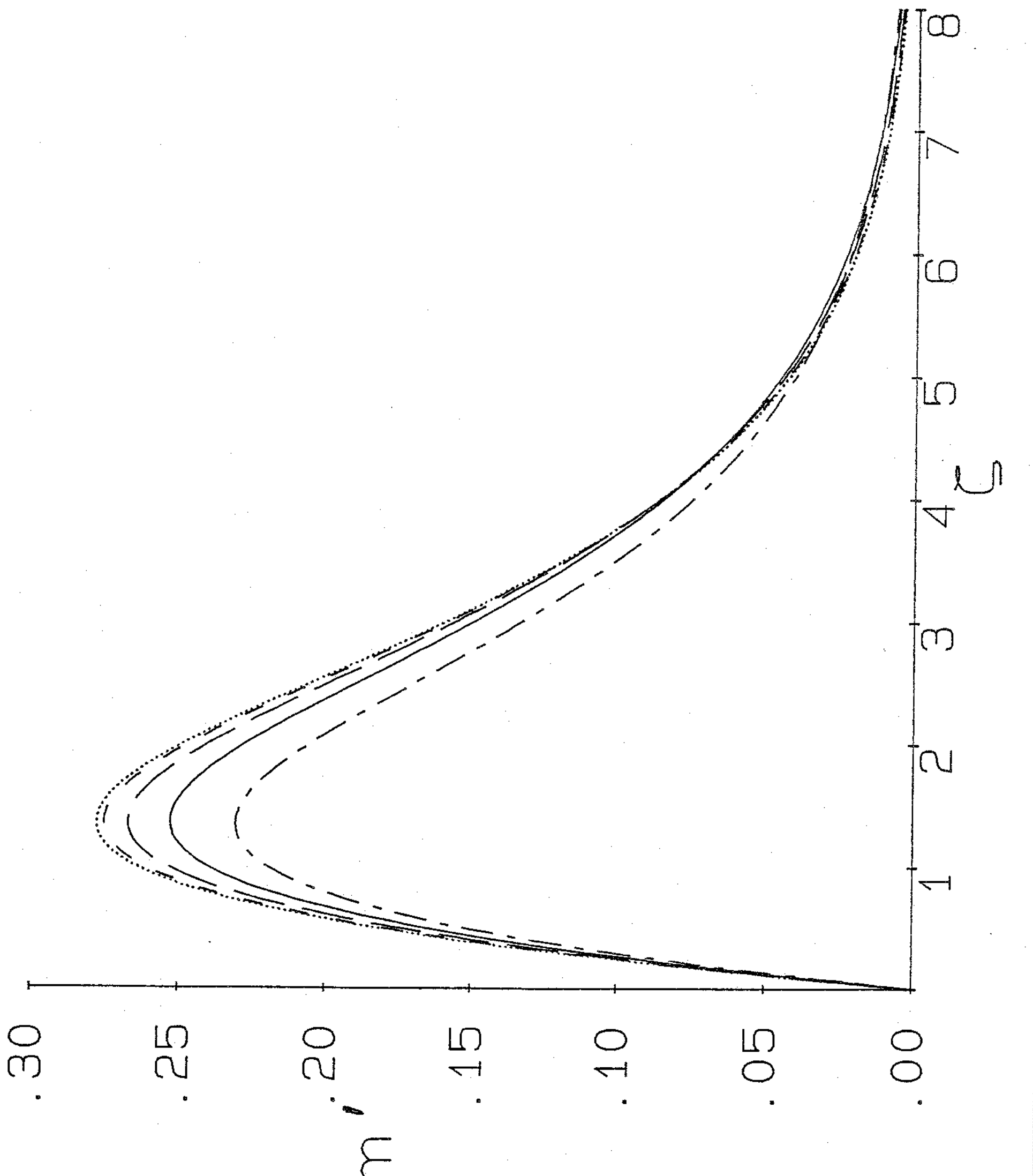


0

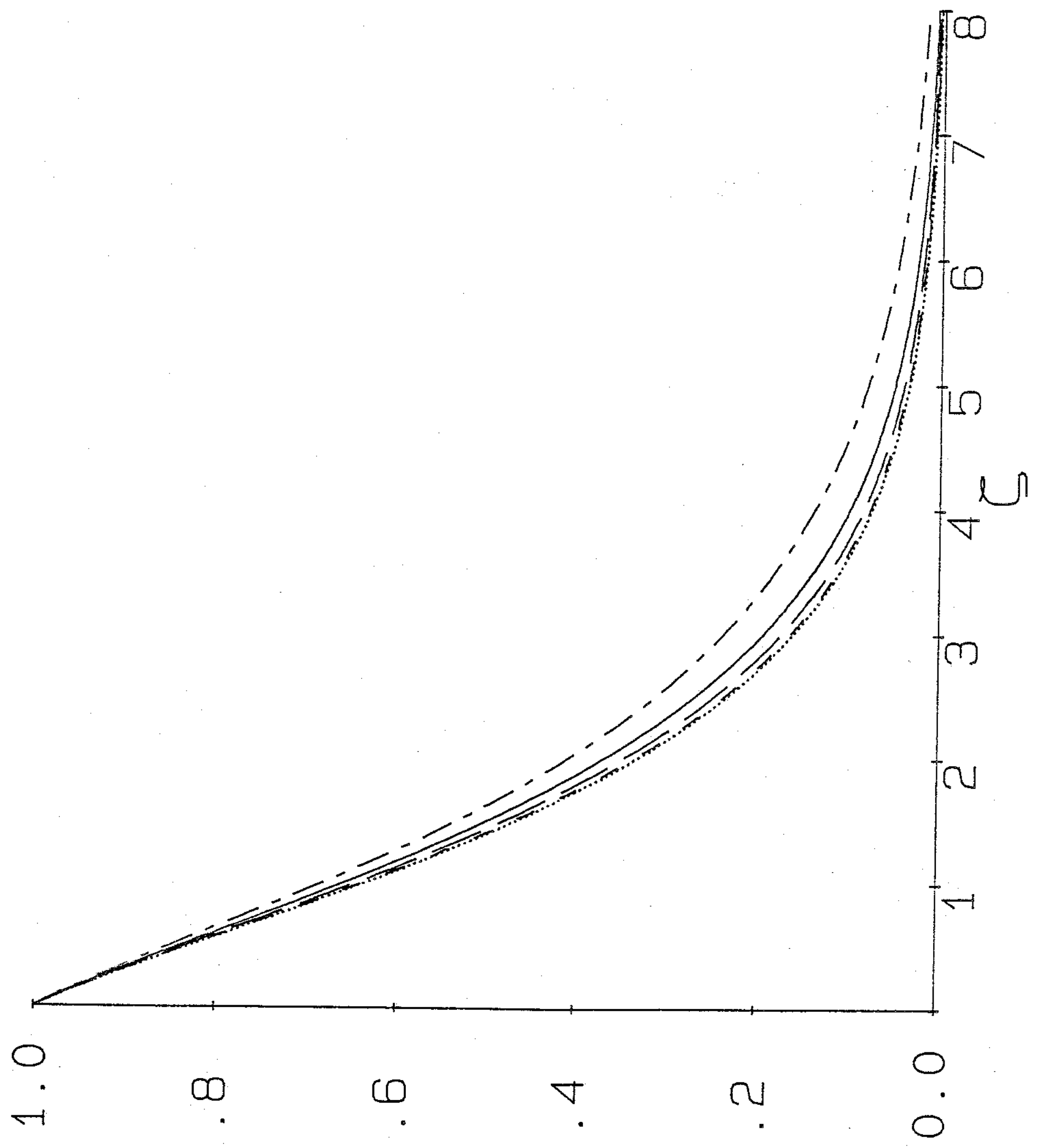

(D) 


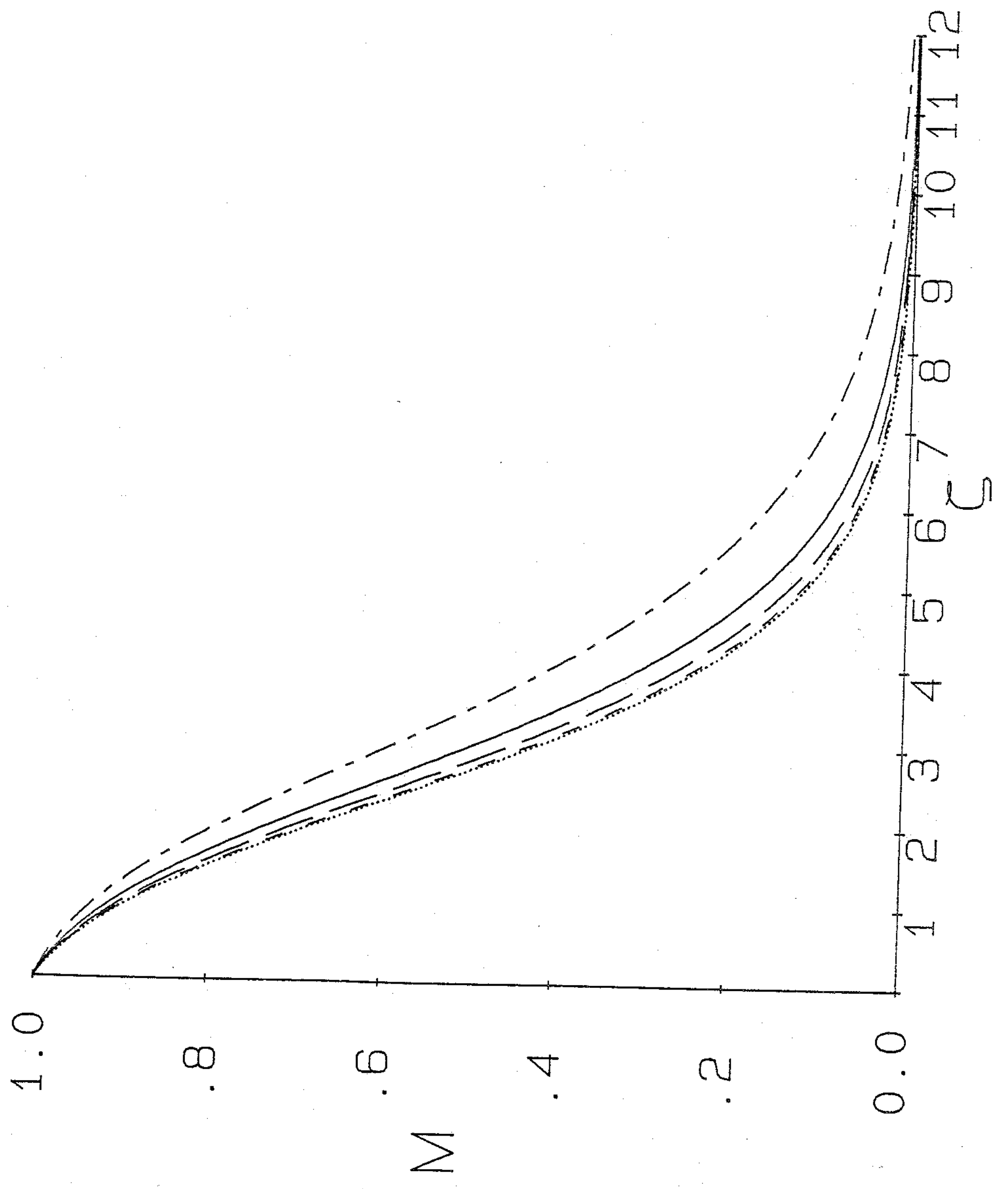


ز

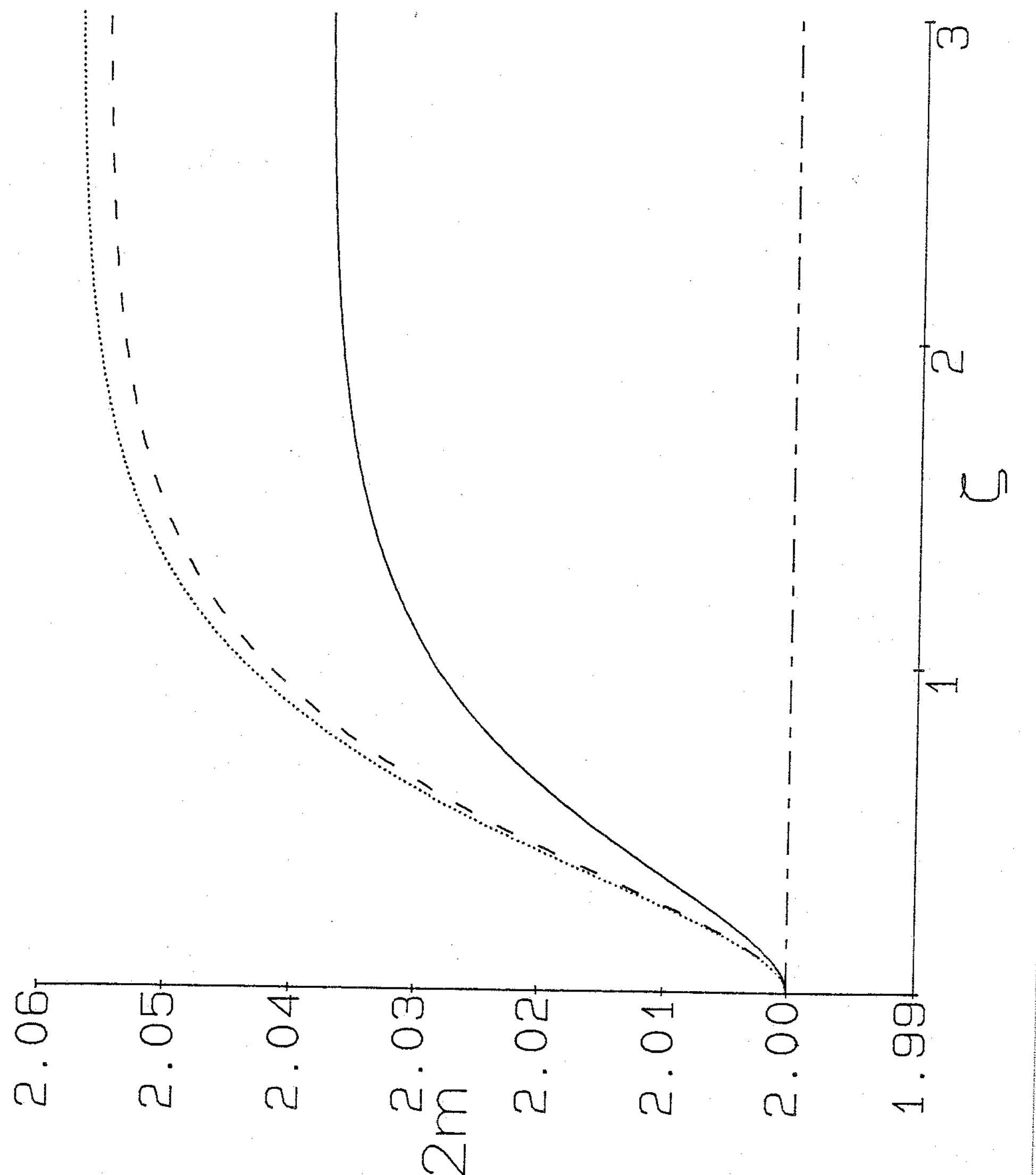




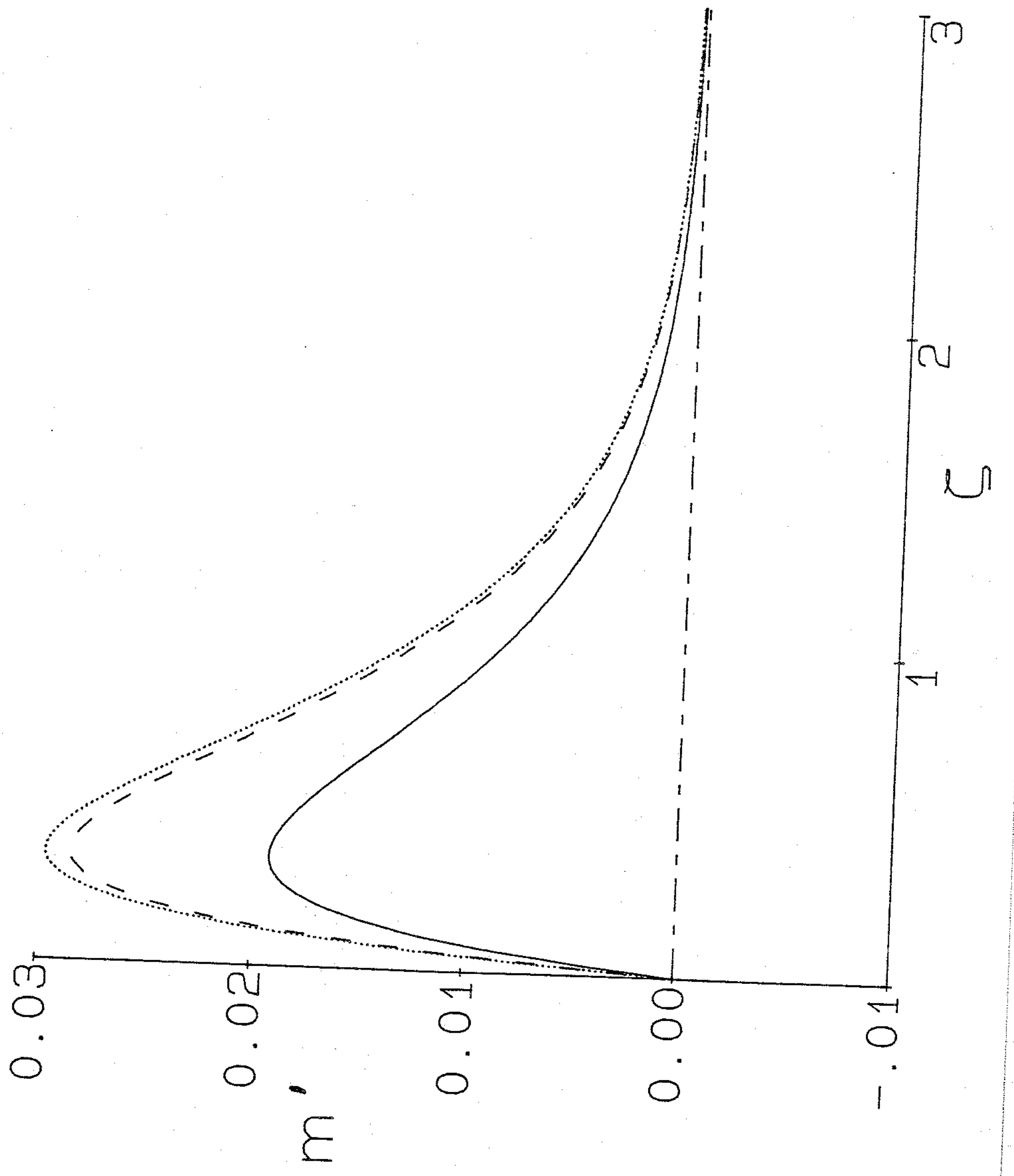

